\title{
Susana Thénon, una voz fuera del cánon`
}

\author{
Manuela Morrone •• \\ Università Ca' Foscari di Venezia \\ Universidad Nacional del Litoral
}

\begin{abstract}
Resumen
Este artículo es un resumen de mi tesis Susana Thénon: una voz disonante en el contexto de la literatura argentina. Susana Thénon fue una poeta, fotógrafa y traductora argentina. Con este trabajo hemos intentado demostrar el genio de esta poeta tan poco conocida tanto en el ambiente literario argentino como italiano, aunque hoy día son muchos los estudiosos que están revaluando su producción literaria y sus poemas son considerados muy actuales en Argentina, especialmente en el contexto de la literatura de género. Thénon prefirió mantenerse alejada del ambiente literario y de las poetas de su época aunque escribió en importantes revistas de poesía, como por ejemplo Agua Viva, Bibliograma, Empresa poética, Encuentro y Sur. Sus poemas son un grito de desesperación y rebelión. A través de los procedimientos estilísticos irónicos, los poemas de Thénon abren grietas en el discurso dominante mostrando lo construido de su esencia. Thénon muestra y destruye todo tipo de categoría cultural y social de género, penetrando en lo más hondo del imaginario colectivo.
\end{abstract}

\section{Palabras clave:}

- feminismo - machismo - Susana Thénon - literatura argentina · poesía de género

\footnotetext{
- Este trabajo es una sintesis de mi tesis, «La poesía de Susana Thénon: una voz disonante en el contexto de la literatura argentina», elaborada y defendida el 11 de marzo de 2016, en el marco del proyecto de doble titulación en Estudios Ibéricos e Iberoamericanos entre la Universidad Ca' Foscari de Venecia y la Universidad Nacional del Litoral.

- Licenciada en Letras en la Univesidad Nacional del Litoral y en Lingue e Letterature europee, americane e postcoloniali en la Universidad Ca' Foscari de Venecia. Se interesa principalmente en la poesía de género argentina. Desde el 2016 colabora en el Portal virtual de la memoria gringa de la FHUC, UNL.
} 


\begin{abstract}
This article is a summary taken from my thesis Susana Thénon, una Voz Disonante en el Contexto de la Literatura Argentina. Susana Thénon was an Argentinian poet, photographer and translator. With this article we have tried to show the genius of this so little-known poet both in the Argentinian literary scene and in the Italian's, even though nowadays many scholars are revaluating her work. Her literary production and her poems are considered to be very up-to-date in Argentina, specially in the context of gender literature.

Thénon preferred to stay away from this social environment and from the poets of her time. However, she wrote in important literary magazines, such as Agua Viva, Bibliograma, Empresa Poética, Encuentro and Sur. Her poems are a cry of despair and rebellion. Through ironical stylistic procedures, Thénon's poems cause rifts in the dominant discourse showing what has been built in its essence. Thénon showed and destroyed all kinds of cultural and social gender categories penetrating deep inside the popular imagination. She did not like the models and categories imposed by a male chauvinist culture.
\end{abstract}

\title{
Key words
}

- chauvinism · feminism - Susana Thénon · Argentine literature · poems · cultural constructions

Susana Thénon fue una poeta, fotógrafa y traductora argentina del siglo XX pero no es tan reconocida en el ámbito literario argentino y mundial. Su figura fue deslizándose del canon literario y siempre se mantuvo a una prudencial distancia del ambiente intelectual de la época. Como nos informa Julián Troksberg ${ }^{1}$, hasta rechazó una beca del CONICET para no «aburguesarse» ${ }^{2}$.

Nació en Buenos Aires el 7 de Mayo de 1935 y murió en la misma ciudad en 1991 de cáncer de cerebro. Era hija única en una familia muy rica de la misma ciudad. Su padre, Jorge Thénon (1901-1985), era un psiquiatra importante de la época; según las fuentes investigadas por Julián Troksberg, el doctor Thénon se carteó varias veces con Sigmund Freud. Además de su profunda devoción al trabajo como psiquiatra, era un militante del Partido Comunista argentino, lo que le costó la pérdida del puesto en el hospital. De lo que confirma la poeta argentina, amiga y compañera de la secundaria de Susana Thénon, Silvia Mazar (1935), la relación entre padre e hija fue siempre muy conflictiva, y esto puede haber tenido consecuencias en sus poemas en los que la figura paterna está casi ausente. La madre, Teodora, era judía y con ella tampoco tuvo una relación fácil, por lo menos hasta la muerte del padre en 1985. En 1954, Thénon se licenció en el Liceo Primero de Señoritas ${ }^{3}$ y eligió estudiar en la Universidad de Buenos Aires ${ }^{4}$, en la que se 
graduó en Letras Clásicas en 1964. A los 18 años trabajó como actriz en El enfermo imaginario (1673) de Molière (1622-1673) e interpretó a la monja en el Rosal de las Ruinas (1921) de Belisario Roldán (1873-1922) que se estrenó en el Teatro Cervantes de Buenos Aires en 1953. Trabajó también como iluminadora en una compañía teatral, lo que le permitió viajar al interior del país. Durante un tiempo fue profesora de latín e investigadora becada del Consejo Nacional de Investigaciones Cientificas y Técnicas de la Argentina. Troksberg, durante una entrevista, nos informó que trabajó también en una revista agraria, en la que se ocupaba de corregir y revisar los artículos. Era contemporánea de Alejandra Pizarnik (1936-1972), pero no hay fuentes que aseguren amistad entre las dos. Lo que sabemos es que ambas publicaron en la Revista Agua Viva y que Thénon colaboró en la dirección de la misma junto al poeta Juan Carlos Martelli ${ }^{5}$. A su vez, fue miembro de la dirección de otra revista de la época, Airon. Su producción poética fue publicada, además de en estas dos revistas, en Bibliograma, Empresa poética, Encuentro y en Sur. La totalidad de su obra se encuentra editada en cinco libros publicados entre 1958 y 1987: Edad sin tregua (1958), Habitante de la nada (1959), De lugares extraños (1967), Distancias (1984) y Ova completa (1987). Durante diecisiete ańos (entre 1967 y 1984) dejó de publicar poemas y se dedicó a la fotografía. Sus fotografías fueron publicadas en formato carpeta por Ediciones Anzilotti (Buenos Aires, 1998) y algunas fueron reproducidas en La morada imposible (tomo 1, 2007 y tomo 2, 2012). Obtuvo el primer premio de fotografía humorística con una obra de la serie «Humor Blanco y Humor Negro» del Concurso «Parisiennes en las Artes». Muchas de sus fotos aparecieron acompañadas por poemas del poeta austríaco Rainer Maria Rilke (1875-1926), traducidos por ella misma al castellano. Durante siete años se dedicó únicamente a fotografiar a la bailarina argentina Iris Scaccheri (1949-2014), que según lo que afirma Mazar, fue, aunque tormentoso, el gran amor de su vida. Registró con su cámara momentos de obras teatrales de distintos dramaturgos en los que Iris bailaba, como por ejemplo, Carmina Burana; Yo odio, yo amo; La muñeca; Juana, reina de Castilla y Aragó; Hosanna; ¿Me quisiste alguna vez?; Homenaje a Dore Hoyer; Temas españoles; La Ascensión y Dos mujeres. (Negroni y Barrenechea en Thénon, 2007: 199).

A pesar del innegable valor de la producción literaria y fotográfica de la poeta no hay muchos estudios en Argentina dedicados a su obra. Lo que se sabe sobre la misma se debe mayormente a dos grandes amigas suyas, Ana María Barrenechea (1913-2010) y María Negroni (1951), quienes se ocuparon de editar póstumamente toda su producción literaria y parte de la fotográfica, y a la poeta argentina Inés Manzano, quien las ayudó en la transcripción de los manuscritos. Fruto de este trabajo de recolección de su obra edita e inédita es el libro, La morada imposible, dividido en dos tomos, el primero publicado en 2001 y el segundo en 2004. En el prólogo del primer tomo María Negroni afirma que Thénon es una poeta «huérfana y sigilosa», porque parece estar unida a aquello que perdió, su voz habla para no decir nada o mejor dicho, para ser la voz de la cosa ausente (Negroni en Thénon, 2007: 13). Y de hecho, quien lee sus poemas tiene la sensación de no ir a ningún lugar, de quedarse en el borde. Susana Thénon podría definirse como una border line, pues no se sentía perteneciente a ninguna generación poética y su estilo heterogéneo no permite asociarla a ningún movimiento literario. Si bien algunas características formales de su producción literaria la relacionan a la generación de los'50 en Argentina, otras con la poesía surrealista y otras con el neorromanticismo. 
La poesía thenoniana propone, grita y destruye las reglas impuestas por el discurso, va más allá de toda construcción e imposición social. Los procedimientos estilísticos utilizados por la poeta se escapan a todas las reglas formales de la lengua hasta alcanzar un lenguaje nuevo, que no cabe en las categorías lingüísticas:

En el fondo, cualquier cosa es cierta o falsa, cuando se convierte en generalización. El lenguaje no se emputece ni se refina ni se alambica ni se simplifica. Es todas esas cosas desde siempre. Que nosotros seamos miopes y «descubramos» por centésima vez las mismas "novedades» no es culpa del lenguaje, sino de una rara, paralizante enfermedad que nos hace decir "trasero» cuando lo único viable es decir «culo», y viceversa... (Thénon, 2004: 220)

Thénon estaba convencida de que lenguaje y pensamiento tenían que ir a la par. El pensamiento casi nunca es ordenado; el tiempo en el que se manifiestan las experiencias en la mente es más rápido y lógicamente confuso con respecto a la realidad. Ella quería un lenguaje capaz de representar estos procesos de la mente.

El lenguaje thenoniano aniquila todo lo pre-construido, sus expresiones son dinamitas para todo tipo de regla sintáctica y léxica, explotan en contra de todo edificio ideológico fijado en los imaginarios colectivos. Este procedimento estilístico típico de la ironía es muy evidente en su último libro Ova completa, por ejemplo en esos versos en los que la poeta denuncia la corrupción de las instituciones sociales, de los cargos públicos: "¿-y qué me va a pasar?/ — depende del comisario de turno/ si le toca a Loiácano/ por ahí la saca barata/ y en menos de unos días está afuera» (Thénon, 2001: 185). Su voz poética es un grito que no encuentra salida, que se choca contra las paredes de los edificios sociales y queda encerrado en la habitación sin puertas ni ventanas de lo inexplicable: «—¿dónde está la salida?/ —iperdón?/ — [...] —no/ no hay salida/ [...] no/ ya es muy tarde/ desde las diez hay entrada prohibida» (Thénon, 2001: 184). No hay salida, no hay alivio ni respuesta: todo se hunde en la condición de fragilidad del hombre moderno, no existen manijas a las que agarrarse. Susana Thénon destruye todos los clichés de su época, incluso aquellos relativos a la sexualidad y las categorías de género. Nunca se definió como escritora feminista, muy de moda en aquel momento, pero tampoco se puede decir que está adentro del canon falocéntrico. Su actitud es, más bien, como la del salmón, en contra de la corriente. No le importa decir lo que piensa, ni crearse enemigos, sus ideas se manifiestan claramente en sus poemas. De acuerdo con Reisz el suyo es un grito exasperado en contra de un feminismo delirante, de un imperialismo norteamericano, de una crítica literaria snob, y sobre todo, de un antimoralismo o un moralismo al revés (Reisz, 1988: 184). 


\title{
Deconstrucción de las categorías de género
}

\author{
De madrugada \\ Ella se tocó las manos. \\ Ella recuerda que nada importa \\ aunque su sombra siga corriendo \\ alrededor de la noche.
}

("Ella»)

Leer los poemas de Susana Thénon es arrojarse a un mar hecho de arena, impalpable, doloroso. El mundo que su mano va representando está hecho de abismos lejanos y aparentes, de espejos que reflejan caras que no existen, «rostros en los atardeceres», las manos no acarician, sino que arañan cuerpos de papel. El sujeto poético de sus poemas está perdido entre las máscaras del tiempo y del espacio, siempre en busca de una identidad que se le desliza entre los dedos.

Los poemas de Susana Thénon actualizan una ruptura con el discurso que desde siempre impone a la mujer un rol subalterno y dependiente del hombre. Alicia Poderti afirma:

Si aceptamos el argumento de Foucalt acerca de que lo «verdadero» depende de quien toma control del discurso, entonces es razonable pensar también que la dominación de los hombres sobre los discursos ha atrapado a las mujeres, durante mucho tiempo, en una red de unas «verdades» masculinas (Poderti, 1995: 70)

En acuerdo a Poderti, estas verdades masculinas imponen división de los géneros sexuales y discriminación de la mujer. Las estrategias irónicas en los poemas de Thénon actúan imponiendo una reflexión y una toma de conciencia de los procedimientos de exclusión de la mujer. Obsérvese por ejemplo el poema «And so are you» de Ova completa:

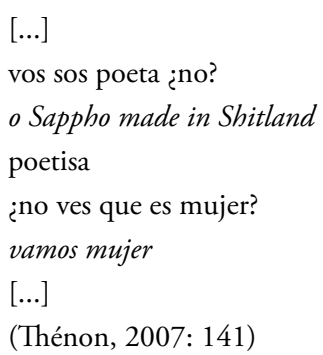

La aclaración irónica «vos sos poeta ¿no?/ [...] poetisa» opera mostrando y ridiculizando la construcción cultural falocéntrica del sustantivo "poetisa». En acuerdo con Gamboa este poema va creando un doble juego a partir de subrayar la percepción de externa de la figura social de la poeta a través de la enunciación del oficio en femenino, desde un punto de vista despectivo. (2013: 6, 7). El sustantivo "poeta» tuvo por mucho tiempo y, en parte, sigue teniendo todavía, una denotación excluyente de género masculino. Según Aldana, Nicanor Parra impuso 
una revolución en la definición del mismo, asociándolo tanto a la mujer como al hombre. Thénon en sus poemas actualizó tal renovación referencial.

[...] Se podría plantear que el impulso revolucionario que Parra introdujo en la poesía, imponiendo una forma de ampliar la concepción que encerraba el sustantivo «poeta», se actualizó en la fuerza corrosiva de los últimos poemas de Thénon, señal del destino de una escritura impiadosa, dispuesta a golpearle los ojos a sus lectores (Aldana, 2001: 2).

Obsérvese también el poema inédito, Las mujeres poetas:

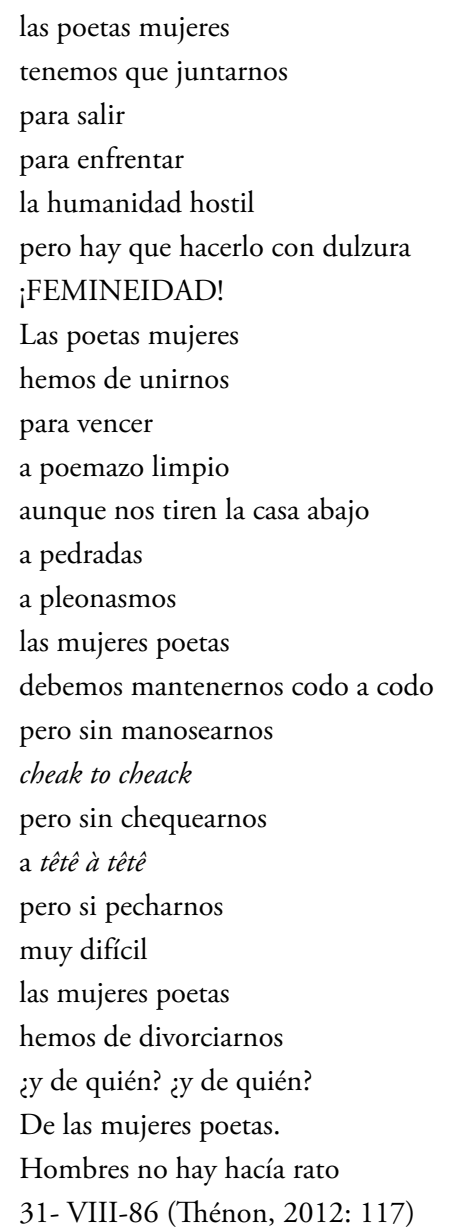

Los juegos de palabras, las interferencias con lenguas extranjeras junto a términos del lenguaje callejero y del tango ("cheak to cheak/ pero sin chequearnos»", "a têtề à têtê /pero sin pecharnos»") actúan creando una con-fusión lingüística y una contradicción discursiva. Barrenechea indicó la contraposición de códigos y registros diversos en los poemas de Thénon como «naturaleza heteroglósica del lenguaje y heterogenérica del discurso».

Toda diferencia a la regla implica discriminación y aniquilación. La mujer, por su carencia fálica, ha sido desde siempre considerada inferior al hombre y, desde Aristóteles a Freud, la tradición relega a la mujer un rol subalterno a los de los 
hombres. Las poesías de Susana Thénon rompen con todas las clasificaciones de género, mostrando su raíz artificial; no hay una mujer que habla ni un hombre, simplemente un ser que codifica en mensajes sus emociones y pensamientos. En acuerdo con Ostrov, la categoría del género propone pensar lo masculino y lo femenino no como correlatos psíquicos naturales del sexo anatómico, sino como construcciones fundamentalmente culturales que interpretarían las diferencias anatómicas (Ostrov, 2004: 16). No sería azaroso suponer que el cuerpo en algunos poemas de Thénon indique no tanto el cuerpo humano de un individuo, sino que sea símbolo del corpus de leyes sociales y culturales que imponen roles sociales de género y discriminación: «Despojémonos de todo aquello/ seguro/ que se proyecta al exterior [...] Despojémonos de todo cuanto/ nos conformó a imagen y semejanza nuestra», (Thénon, 1958: 29). El uso del modo imperativo del verbo «despojar» opera como exhortación a la de-construcción de la «imagen y semejanza nuestra». En acuerdo con Ostrov, la materialidad corporal -el cuerpo como materiaconformaría una instancia lingüísticamente establecida, constituida como efecto de un proceso de materialización llevado a cabo por y a través del lenguaje (2004: 18). Las construcciones sociales de género y del estereotipo de hombre como ser superior y dominante y de la mujer como creatura frágil es evidente en el poema VI de Edad sin tregua:

La rosa
perdida
de sexo ligero,
el tifón
del borracho
en el mar
del asfalto,
socorro,
aledaños
del sol,
que estoy solo
a mi lado
(Thénon, 2007: 39)

«La rosa perdida/ de sexo ligero» remite a la figura de la mujer débil y superficial construida por siglos de paradigmas de escritura falocéntrica. «El tifón/ del borracho» simboliza el hombre según los atributos de fuerza y poder, siendo el tifón una fuerza destructiva. La incomodidad que la instancia poética advierte por estar encasillada en el rol social de la mujer es evidente en el poema "Ahora» de Edad sin tregua:

La vida es esta cosa doméstica que manoseo todos los días con indiferencia, con la pasividad de un ave de corral, sin sueños.

La vida no tiene ese color que se presiente de lejos, 


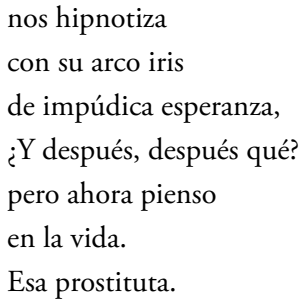

Thénon trata de los prejuicios relativos a la figura de la mujer, encerrada en casa y que trascurre sus días en la indolencia, pasividad e «indiferencia» de las tareas de la casa. El modelo de la mujer como figura relegada a las actividades domésticas se construyó en el siglo XIX. Este prototipo de mujer pasa su días en casa, mirando a la vida detrás de una reja como lo que Adriana Crolla, define como «ave de corral ${ }^{8}$ a la que se asocia el acto de «ver/ mirar». De acuerdo con Crolla esta imagen define un estadio femenino caracterizado por el sometimiento a la tradición masculina y a la perpetuación del papel que la sociedad le asignó (Crolla, 2010: 19). Según la crítica, «el espacio privado a las que fueron confinadas las mujeres a lo largo de los siglos (el gineceo, la habitación, la cocina, la casa) les brindaba protección y las salvaba de los "perversos efectos" del afuera, al mismo tiempo las auto-recluyó y las condenó a su propia auto-inconsciencia». (Crolla 2010: 17). Por lo que la presentación de los dos estereotipos ponen en acto una reflexión sobre la imagen de la mujer como ser «de sexo ligero» construida por la cultura falocéntrica, confinada en casa como «ave de corral».

Thénon deconstruye el significante cuerpo que siempre ha sido interpretado y definido por la cultura en función de determinados rasgos anatómicos excluyentes para la identificación de cuerpo-mujer y cuerpo-hombre. En acuerdo con Laura Scarano el cuerpo es una ficción culturalmente operante, porque nunca se piensa en el cuerpo como algo separado de su género, nunca se ven cuerpos sino estamos acostumbrados a ver hombres y mujeres con cuerpos que representan esa condición sexuada (2007: 42). En el poema «No» del libro El habitante de la nada se muestra y se niega la «jaula» en la que está confinada la mujer:

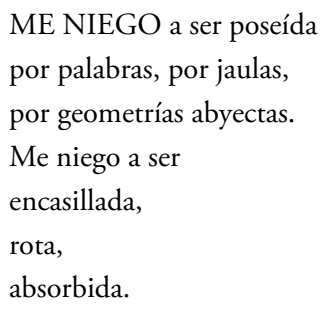

(Thénon, 1959: 52)

La poeta va volcando el rol de la mujer «encasillada», cumpliendo lo que Juan Mukarovsky define como la "función estética» de la poesía (Scarano, 2007: 72). De hecho el lenguaje poético thenoniano no es «estéticamente indiferente» (Scarano, 2007: 72), sino que tiene la función social de mostrar lo construido de la imagen de hombres y mujeres tanto desde una mirada falocéntrica como feminista. 
Susana Thénon rompe el vidrio de las apariencias relativas a la imagen de la mujer-poeta enferma, deprimida, alcohólica, anoréxica típica de los años '70 y grita en contra de estos estereotipos. Obsérvese el poema «La antología» perteneciente al libro Ova completa:

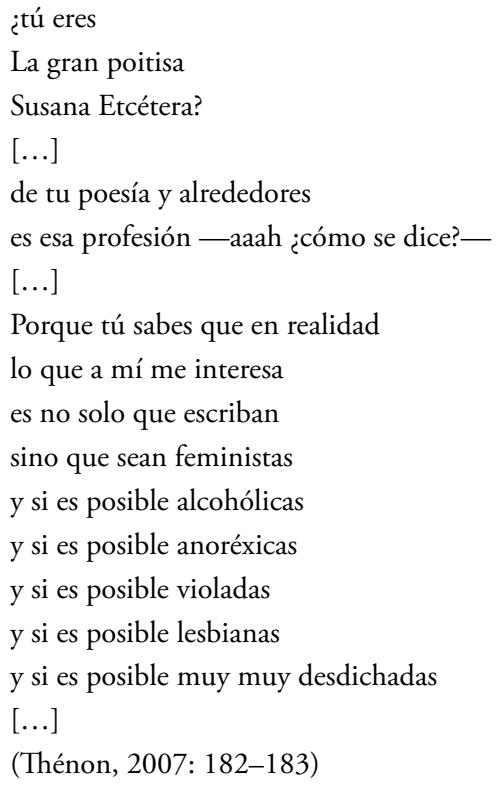

La ironía, en este poema, golpea a la puerta de todo lo construido para escarnecerlo y destruirlo; el suyo es un ataque a la crítica y a los editoriales de la época que venden e incrementan la proliferación de modelos de las poetas-mujeres ("la poitisa»).

Susana Thénon crea, a través del lenguaje, un yo poético que se desliza en contra de todo lo pre-construido, buscando una voz propia, que no es el recuerdo de algo preexistente. Desde los versos de sus poemas se dibuja un alma pura, que no puede ser definida ni imaginada, por ello afirma que nuestra imaginación está forjada "por dientes y espejos» (Thénon, 2007: 27) que no nos pertenecen por naturaleza, sino que heredamos culturalmente. Este ser puro, por el contrario, no tiene ninguna herencia, se escapa de todo esto, vive en un estado de sobrevuelo constante.

Con este trabajo nos proponemos despertar el interés y colaborar en el conocimiento de la obra de esta poeta que se está volviendo siempre más actual hoy en día, especialmente en el contexto de la poesía de género. Su voz se hace escuchar, rompe los versos, rasguña las páginas y atrapa los pensamientos. El suyo es un grito de desesperación escondido detrás de una ironía amarga, sus poemas son las dos caras de la luna: su estilo tan perturbador esconde una verdad desconcertante, la de un mundo de reglas e imposiciones culturales impuestas por una tradición falologocéntrica. Los poemas de Thénon destruyen todo edificio cultural y social preconstruido; su sensibilidad revolucionaria y su genio increíble, justifican que alcance la visibilidad que merece. 


\section{Notas}

${ }^{1}$ Julián Troksberg es director de una serie de documentales, Biografias de la literatura, para el canal Encuentro (2015-2016). Produjo un documental sobre Susana Thénon, Biografias de la literatura / Susana Thénon, que se presentó en los últimos meses de 2016.

${ }^{2}$ Según información brindada por Alicia Parodi, amiga de la poeta, Troksberg tomó contacto con poetas y amigos de Susana Thénon.

${ }^{3}$ Actualmento Liceo $n^{\circ} 1$ "José Figueroa Alcorta", Av. Santa Fe 2778, Buenos Aires.

${ }^{4}$ Cuya dirección era Viamonte 440, Buenos Aires.

${ }^{5}$ Juan Carlos Martelli fue un escritor argentino (1934-2008) que además de haber sido director de la Revista Agua viva, dirigió también la revista Casos y La Revista de los Jueves de Clarín. Fue codirector de la revista Diners (junto a Miguel Brascó) y de la revista-libro Cuadernos de Mr. Crusoe (junto a Horacio Verbitsky). Datos extraídos de «Murió el escritor Juan Carlos Martel», Agencia Telám, Revista online La voz, Viernes 18 de abril de 2008) http://archivo.lavoz.com.ar/nota.asp?nota_id=181630 (Consultado el 23/12/2015)

${ }^{6}$ Las voces «checar" $\mathrm{y}$ "chequear», creadas por influjo del inglés «to check» "comprobar», son correctas y equivalentes con el significado de «someter, algo o a alguien, a examen, control o verificación", voy a checarme-chequearme la presión; hay que checar-chequear la graduación de los lentes. (BETA Academia mexicana de la lengua) http://www.academia.org.mx/ espin/Detalle?id=98 (Consultado el 17/01/2016).

7 Pechar: Pedir prestado algo, particularmente dinero (TC.); solicitar (LCV.); sablear// empujar, arremeter// embestir con el pecho una persona o caballo; empujar o dar con el pecho (LS.). Ricardo García Blaya, Diccionario del Lunfardo Todo tango, http://www.todotango.com/ buscar/?kwd=pechar (Consultado el 17/01/2016)

${ }^{8}$ Expresión usada por la misma Thénon en el poema "Ahora".

\section{Referencias bibliográficas}

\section{Sobre género}

AA:VV (1995). Para leer la literatura argentina, Escrituras, cuerpos y sexualidad, en Tramas (Vol. I, n. 2). Córdoba: Ediciones del Caminante. Bemberg, M.L. (1972). El mundo de la mujer. [Recuperado en línea]. Consultado el 13 de enero de 2016 en https://vimeo.com/14196200.

Crolla, A. y Vallejos, O (2010). Estudios comparados de la literatura actual. Santa Fe: Ediciones UNL.

Femenías, M.L. (2000). Sobre sujeto y género, lecturas feministas desde Beauvoir a Butler. Buenos Aires: Editorial Catálogos.

Genovese, A. (1998). La doble voz, poetas argentinas contemporáneas. Buenos Aires: Editorial Biblos.

Lipovetsky, G. (1999). La tercera mujer. Barcelona: Editorial Anagrama. Lopez GiL, M. (1999). El cuerpo, el sujeto, la condición de mujer. Buenos Aires: Editorial Biblos.

Morgade, G. (2001). ¿Existe el cuerpo... (sin el género)?. En Sexualidad 
y educación, Ensayos y experiencias (38), año 7, Buenos Aires: Ediciones Novedades Educativas.

Ostrov, A. (2004). El género al bies, cuerpo, género y escritura en Cinco narradoras latinoamericanas. Córdoba: Alción Editora.

Piña, C.; Alvarez, M.A.; García, A.M.; Jara, S.; Moure, C. (1997). Mujeres que escriben sobre mujeres (que escriben). Buenos Aires, Ediciones Biblos. Poderti, A. (1995). Una literatura del noroeste: escritura femenina, cuerpo y discurso Escritura, Cuerpo y sexualidad, en Tramas 1(2), Córdoba: Ediciones el Caminante.

Scarano, L. (2010). Sermo intimus, modulación histórica de la intimidad en la poesía española. Mar de Plata: Editorial de la Universidad Nacional de Mar de Plata.

(2007). Palabras en el cuerpo, Literatura y experiencia. Buenos Aires: Editorial Biblos.

\section{Diccionarios}

Academia mexicana de la lengua, Beta [en línea]. Consultado el 17 de enero de 2016 en http://www.academia.org.mx/espin/Detalle?id=98. García Blaya Ricardo, Dicionario del Lunfardo, Todo tango [Recuperado en línea]. Consultado el 17 de enero de 2016 en http://www. todotango.com/buscar/?kwd=pechar.

\section{Bibliografía específica de y sobre la obra de Susana Thénon} Aldano, F. (2001). El caos preparado como una fiesta, en La morada imposible, de Susana Thénon, (294), Buenos Aires: Ediciones Corregidor [Recuperado en línea]. Consultado el 18 de noviembre de 2016 en file://C:/Users/Utente/Desktop/bazar\%20(1).pdf.

Barrenechea, A.M. (2015). Metamorfosis de espacios, acontecimientos y sujetos textuales en un poema de Susana Thénon. En Elpuente de las palabras. Homenaje a David Lagmanovich, (n. 50). Buenos Aires: Collection Interamer [Recuperado en línea]. Consultado el 12 de enero de 2016 en http://www.educoas.org/Portal/bdigital/contenido/interamer/interamer_50/az_barre.aspx?culture=en

- (2007). Génesis de tres distancias de Susana Thénon. Alicante: Biblioteca Virtual Miguel de Cervantes.

- (1997). Susana Thénon y su subversión del canon. En Atípicos en la literatura latino-americana, Universidad de Buenos Aires: Oficina de publicación del C.B.C.

Thénon, S. (2012). La morada imposible. Tomo 2. Buenos Aires: Ediciones Corregidor.

(2007). La morada imposible. Tomo 1. Buenos Aires: Ediciones Corregidor.

\section{Morrone, Manuela}

